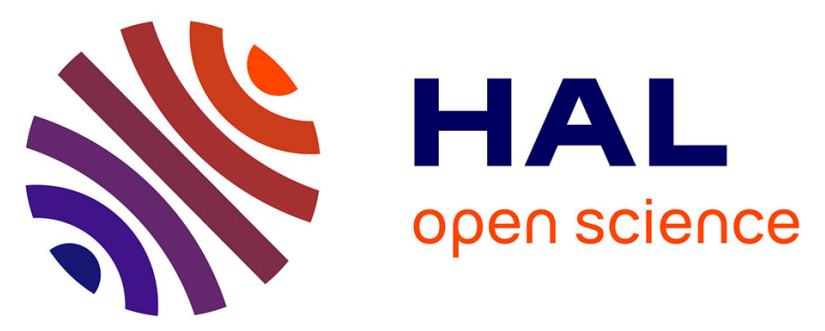

\title{
Interference effects and Stark broadening in XUV intrashell transitions in aluminum under conditions of intense XUV free-electron-laser irradiation
}

Eric Galtier, Frank Rosmej, Annette Calisti, Bernard Talin, Caroline Mossé, Sandrine Ferri, Valery Lisitsa

\section{To cite this version:}

Eric Galtier, Frank Rosmej, Annette Calisti, Bernard Talin, Caroline Mossé, et al.. Interference effects and Stark broadening in XUV intrashell transitions in aluminum under conditions of intense XUV free-electron-laser irradiation. Physical Review A : Atomic, molecular, and optical physics [1990-2015], 2013, 87, pp.033424. 10.1103/PhysRevA.87.033424 . hal-00825989

\section{HAL Id: hal-00825989 \\ https://hal.science/hal-00825989}

Submitted on 27 May 2013

HAL is a multi-disciplinary open access archive for the deposit and dissemination of scientific research documents, whether they are published or not. The documents may come from teaching and research institutions in France or abroad, or from public or private research centers.
L'archive ouverte pluridisciplinaire HAL, est destinée au dépôt et à la diffusion de documents scientifiques de niveau recherche, publiés ou non, émanant des établissements d'enseignement et de recherche français ou étrangers, des laboratoires publics ou privés. 


\title{
Interference effects and Stark broadening in XUV intra-shell transitions in aluminum under conditions of intense XUV Free Electron Laser irradiation
}

\author{
E. Galtier* \\ SLAC National Accelerator Laboratory, 2575 Sand Hill road, Menlo Park, California 94025, USA
}

\author{
F. B. Rosmej ${ }^{\dagger}$
}

Sorbonne Universités, Pierre et Marie Curie, UMR 7605, LULI, case 128, 4 Place Jussieu, F-75252 Paris Cedex 05, France

A. Calisti, B. Talin, C. Mossé, and S. Ferri

PIIM, UMR 7345, Aix-Marseille Université, Centre St. Jérôme, 13397 Marseille, Cedex 20, France

\section{S. Lisitsa}

Russian Research Center "Kurchatov", Kurchatov Square, 46, Moscow 123182, Russia and Moscow Institute of Physics and Technology, 141700 Dolgoprudny, Russia

(Dated: February 15, 2013)

\begin{abstract}
Quantum mechanical interference effects in the line broadening of intra-shell transitions are investigated for dense plasma conditions. Simulations that involved LSJ-split level structure and intermediate coupling discovered unexpected strong line narrowing for intra-shell transitions L-L while M-L transitions remained practically unaffected by interference effects. This behaviour allows a robust study of line narrowing in dense plasmas. Simulations are carried out for XUV transitions of aluminum that have recently been observed in experiments with the FLASH Free Electron Laser irradiating solid aluminum samples with intensities greater than $10^{16} \mathrm{~W} / \mathrm{cm}^{2}$. We explore the advantageous case of $\mathrm{Al}$ that allows first, simultaneous observation of $\mathrm{M}-\mathrm{L}$ transitions and $\mathrm{L}-$ L intra-shell transitions with high resolution grating spectrometers and, second, has a convenient threshold to study interference effects at densities much below solid. Finally we present simulations at near solid density where the line emission transforms into a quasi-continuum.
\end{abstract}

PACS numbers: $32.60 .+\mathrm{i}, 41.60 . \mathrm{Cr}, 32.70 . \mathrm{Jz}, 32.80 . \mathrm{Hd}$

\section{INTRODUCTION}

In the past few years, technological progresses allowed to make available to the scientific community the $4^{\text {th }}$ generation light sources, namely the Free Electron Lasers (FELs). The FEL is a unique source of light: $10^{12}$ photons per pulse, photon energy in the XUV/X-ray range, pulse duration down to femtosecond time scale, high repetition rate $(10-120 \mathrm{~Hz})$ and easily tunable photon energy. The outstanding combination of these parameters has initiated a world-wide interest to FEL's in planetary science [1], astrophysics [2], inertial confinement fusion [3], high energy density physics [4] and to study exotic states of matter never created in laboratories so far $[5,6]$.

As the plasma frequency of XUV photons is above solid density, the FEL radiation penetrates deep into the solid and allows therefore volumetric heating (in contrast to optical laser radiation that is absorbed at the surface critical density much below solid). At intensities larger than about $10^{16} \mathrm{~W} / \mathrm{cm}^{2} \mathrm{XUV} / \mathrm{X}$-ray FEL radiation may deplete completely certain electronic states via photoion-

* egaltier@slac.stanford.edu

$\dagger$ Also at LULI, École Polytechnique, CEA, CNRS, Physique Atomique dans les Plasmas Denses PAPD, Route de Saclay, F91128 Palaiseau Cedex, France ization $[7,8]$. As the pulse duration is of the order of the autoionization rate, hollow ion states are formed via successive photoionization [5], and solid matter can practically instantaneously transformed into an exotic state of matter: a hollow crystal $[6,9]$. Although photoionization is a well understood energy deposition mechanism, the mechanisms of subsequent energy equilibration and matter heating that induced the transition from solid to Warm Dense Matter to strongly coupled dense plasma is not well explored.

Under such conditions high resolution XUV/X-ray spectroscopy (gratings and Bragg crystals) is an important method to obtain plasma information independent of simulation. In particular, it permits to study the disintegration of crystalline order and material heating as has been demonstrated recently [9]: electron densities $n_{e}$ of about $10^{22}-10^{23} \mathrm{~cm}^{-3}$ and electron temperatures of $\mathrm{T}_{e} \approx 30 \mathrm{eV}$ have been inferred from the analysis of intensities of M-L and L-L transitions in multiple excited aluminum ions. This analysis allowed also to qualitatively infer the temporal evolution of the heated sample (disintegration of crystalline order).

In the present work, we investigate the complex line broadening of L-L intra-shell transitions to make use of the information contained in the contour shape of the M$\mathrm{L}$ and $\mathrm{L}-\mathrm{L}$ transitions of $\mathrm{Al} \mathrm{III} \mathrm{and} \mathrm{Al} \mathrm{IV}$ that corresponds to the emission of the type $K^{2} L^{7} M^{m} \rightarrow K^{2} L^{8} M^{m-1}+h \nu$ 
and $K^{2} 2 s^{1} 2 p^{6} M^{m} \rightarrow K^{2} 2 s^{2} 2 p^{5} M^{m}+h \nu$ with $m=2$ and 1. Detailed calculations are carried out with the PPP code $[10,11]$, that provides fast and accurate line profiles in plasmas for very complex transition arrays. PPP accounts for all the main mechanisms of line broadening: lifetime broadening due to spontaneous emission, Stark broadening. Particular attention is payed to interference effects [12] which turned out to be a major player for the contour shape analysis [13, 14].

\section{INTERFERENCE EFFECTS IN LINE BROADENING THEORY}

The line shape function in the radiative dipole approximation is related to the imaginary part of the Fouriertransformed dipole autocorrelation function. This can be written as a normalized Liouville space-matrix element of the response function:

$$
I(\omega)=I m \ll D^{+}|G(\omega)| D \rho_{0} \gg,
$$

where $\rho_{0}$ is the equilibrium density matrix operator for the active quantum system and $D$ is the dipole operator for the emitting quantum system. The response function, $G(z)$, is given by the one-sided Fourier transform of the bath-averaged evolution operator of the emitter $U(t)$,

$$
\begin{aligned}
G(z) & =i \int_{0}^{+\infty} U(t) e^{-i z t} d t \\
& =(z-L)^{-1} .
\end{aligned}
$$

$L$ is the Liouville operator for the emitter evolution alone. If the interaction fluctuations or collisions are random, a stochastic Liouville equation (SLE) must be solved to obtain $U(t)=<U_{l}(t)>_{l \in L}$ in order to deduce $G(z)$ :

$$
\begin{aligned}
\frac{d U_{l}(t)}{d t} & =-i L(t) U_{l}(t) \\
U_{l}(0) & =1
\end{aligned}
$$

where $l$ is a given time-dependent function of the functional space $L$. In Eq. 3, the Liouville operator is given by: $L(t)=L_{0}+L_{l}(t)$, where $L_{0}$ represents the full complex atomic system of the unperturbed atom and the Stark effect is represented by $L_{l}(t)=-\frac{1}{\hbar} D \cdot E_{l}(t)$ where the electric microfield is created by both surrounding electrons and ions, i.e, $E_{l}(t)=E_{e, l}(t)+E_{i, l}(t)$.

This SLE can be solved either exactly or to a good approximation, only in a few well-known cases. For example, an analytical solution is obtained for the impact limit in which short and rare binary collisional events occur between emitters and perturbers and the mean time between collisions is much longer than the collision time. The second example concerns the static limit where the perturbing ion microfields, acting on emitters, are constant during the radiative process and are well characterized by a probability density. In most of the theoretical models of spectral line shapes in plasmas [15], the time dependence of the perturbation is eliminated, resulting in a spectral line shape that has pure homogeneous and inhomogeneous contributions and that is described by a simple sum of independent electron-impact broadened static components. In this paper, the PPP code developed to provide line profiles for arbitrary atomic systems in a plasma for a large range of conditions, has been used, assuming that the plasma and atomic structure parameters are such that the static ion micro field approximation and the model of a binary collision electronic operator are valid. In this case the quantum-emitter system evolution operator in Eq. 1 contains in the Liouville operator a non-Hermitian, homogeneous electron-impact broadening contribution, which is numerically averaged over the ion microfield interaction with a stationary-field probability distribution $[16,17]$. This procedure yields to a spectral line-shape function that can be written as a sum of rational fractions or generalized Lorentzian spectral components of the line, characterized by a complex frequency and intensity (they are complex, because the non-Hermitian collision operator is used to describe the impact electrons). The electronic collision operator is calculated in the framework of a binary collision relaxation theory. An element of this operator is written as the sum of three terms [18]:

$$
\begin{aligned}
\Phi_{\alpha \alpha^{\prime} \beta \beta^{\prime}} & =\sum_{\alpha^{\prime \prime}} \delta_{\beta \beta^{\prime}} \vec{d}_{\alpha \alpha^{\prime \prime}} \cdot \vec{d}_{\alpha^{\prime \prime} \alpha^{\prime}} G\left(\Delta \omega_{\alpha^{\prime \prime} \beta}\right) \\
& +\sum_{\beta^{\prime \prime}} \delta_{\alpha \alpha^{\prime}} \vec{d}_{\beta^{\prime} \beta^{\prime \prime}} \cdot \vec{d}_{\beta^{\prime \prime} \beta} G\left(-\Delta \omega_{\alpha \beta^{\prime \prime}}\right) \\
& -\vec{d}_{\alpha \alpha^{\prime}} \cdot \vec{d}_{\beta^{\prime} \beta}\left[G\left(\Delta \omega_{\alpha \beta^{\prime}}\right)+G\left(-\Delta \omega_{\alpha^{\prime} \beta}\right)\right]
\end{aligned}
$$

with $\Delta \omega_{\alpha \beta}=\omega-\omega_{\alpha \beta}, \omega_{\alpha \beta}$ being the frequency difference between the states $\alpha$ and $\beta$.

The two first terms are sums over the perturbing states $\alpha^{\prime \prime}$ and $\beta^{\prime \prime}$. If $\alpha=\alpha^{\prime}$ and $\beta=\beta^{\prime}$, they are diagonal and they couple a transition $\alpha-\beta$ with itself, they correspond to the atomic scheme shown on Fig. 1a.

The extra-diagonal terms corresponding to the atomic scheme on Fig.1b appear to be negligible. The third term which is also extra-diagonal, represents the interference effect between the subsets $\alpha$ and $\beta$. It couples a transition $\alpha-\beta$ together with a different transition $\alpha^{\prime}-\beta^{\prime}$. A schematic representation is given on Fig.1c. If these terms are negligible, the collisional operator is diagonal and the interaction operator accounting for ionic Stark effect in the working-subspace $\mid \alpha \beta \gg$ is block-diagonal, reducing the working matrices and making the final calculation easier. The profile is then a sum of ionic Stark components broadened homogeneously by electrons. When it is impossible to neglect extra-diagonal terms, calculations can become very cumbersome. In general terms, their effects on the spectral line shape is a reduction of the electronic line width due to the mixing between the involved radiative transitions.

The function $G(\omega)$ depends on the density, $\mathrm{n}_{e}$, and temperature, $\mathrm{T}_{e}$, of the plasma and is calculated to second order in the radiation-electron interaction. In this 


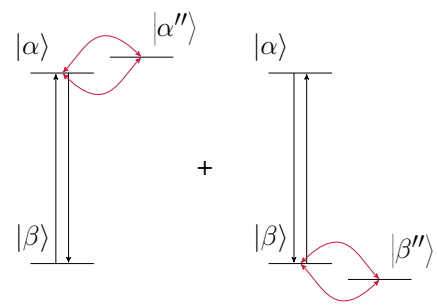

(a)

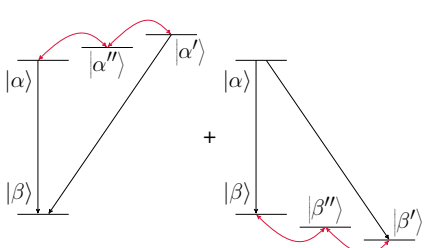

(b)

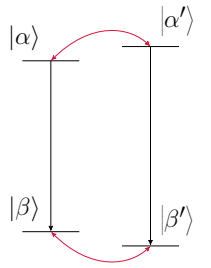

(c)
FIG. 1. Atomic schemes corresponding to: 1a diagonal terms of electronic broadening operator, 1b extra-diagonal terms of electronic broadening operator and $1 c$ interference terms.

paper an expression for $G(\omega)$ based on a modified semiclassical model, in which a strong-collision term $C$ is added to the semi-classical term [19], is used:

$G(\omega)=-\frac{4 \pi}{3}\left(\frac{2 m}{\pi k_{B} T_{e}}\right)^{\frac{1}{2}} n_{e}\left(\frac{\hbar}{m}\right)^{2}\left(C+\frac{1}{2} \int_{y}^{\infty} e^{-x} \frac{1}{x} d x\right)$

where $y$ is given by:

$$
y=\left(\frac{\hbar n^{2}}{2 z}\right)^{2} \frac{\omega^{2}+\omega_{p}^{2}+\omega_{\alpha \alpha^{\prime \prime}}^{2}}{E_{H} k_{B} T_{e}} .
$$

In this expression, $n$ is the principal quantum number of state $\alpha, z$ is the charge of the ionic core, $\omega_{p}$ is the plasma frequency and $E_{H}$ is the ionization energy of atomic hydrogen. An impact parameter cutoff at $b=v / \omega_{\alpha \alpha^{\prime \prime}}$ has been introduced in order to take into account that the integrand of the thermal average contains oscillating exponentials of the frequency difference $\omega_{\alpha \alpha^{\prime \prime}}$ in case of nondegenerate systems.

In the following, the impact limit expression $G(\omega=0)$ has been taken, in order to accelerate the line shape calculations [10]. We have checked this approximation for the transitions originating from the configurations $K^{2} L^{7} M^{1}$ and $K^{2} L^{7} M^{2}$ in aluminum, of particular interest in this paper, and found negligible difference when the frequency dependence has been taken into account. Fig. 2 shows the contributions of the electronic broadening operator to the spectral distribution of $K^{2} L^{7} M^{2}$ configuration for $T_{e}=25 \mathrm{eV}$ and $n_{e}=2 \times 10^{22} \mathrm{~cm}^{-3}$ accounting (blue line) or not (red line) for the frequency dependence. Calculations have been done without taking into account interference terms.

Spectral line shape calculations with the PPP-code require the knowledge of atomic data such as radiator energy levels and reduced dipole matrix elements.

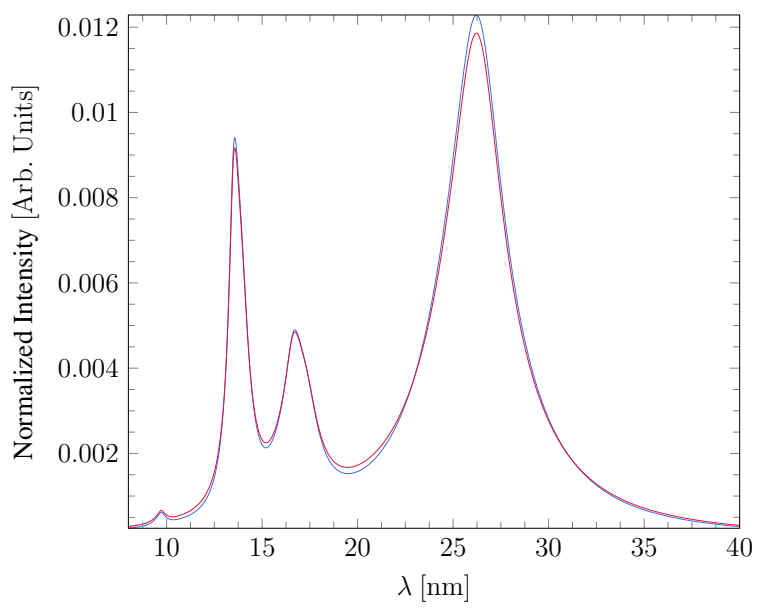

FIG. 2. Electronic broadening effect on the spectral distribution of $K^{2} L^{7} M^{2}$ configuration for $\mathrm{T}_{e}=25 \mathrm{eV}$ and $\mathrm{n}_{e}=2 \times 10^{22} \mathrm{~cm}^{-3}$ accounting (blue line) or not (red line) for the frequency dependence. Curves have been normalized to their own integral.

They have been calculated with the relativistic multicongurational atomic code FAC [20] including LSJsplit level structure and intermediate coupling. The stationary-field probability distribution is obtained by the APEX model $[16,17]$. Particular attention has to be paid to non-LTE populations (dynamic line shapes) and atomic structure of the $K^{2} L^{7} M^{1}$ and $K^{2} L^{7} M^{2}$ configurations. For the M1-configurations, Boltzmann like populations are achieved for electron densities above $n_{e}$ $=10^{19} \mathrm{~cm}^{-3}$ because radiative decay rates are only of the order $A=10^{9}-10^{10} \mathrm{~s}^{-1}$ and these states are not autoionizing. Therefore LTE-populations are well justified for all densities considered in this paper. For the $K^{2} L^{7} M^{2}$ configurations the situation is more complex as these states are autoionizing. Detailed atomic structure calculations show that these states are not only coupled to the ground state $K^{2} L^{8}$ with autoionizing rates of the order of $\Gamma=10^{12} \mathrm{~s}^{-1}$, but also to excited states $K^{2} L^{7} M^{1}$ with huge autoionizing rates being of the order of $\Gamma=$ $10^{13}-10^{15} \mathrm{~s}^{-1}[6,21]$. Therefore, LTE-populations can only be achieved for electron densities above solid density and non-LTE level populations have to be considered for almost all parameters of practical interest. However, it has been shown recently [22] with detailed collisionalradiative population kinetics involving all LSJ-split autoionizing levels that excited states coupling effects drive the autoionizing states $K^{2} L^{7} M^{2}$ to Boltzmann populations for densities larger than only $\mathrm{n}_{e}=10^{21} \mathrm{~cm}^{-3}$. This indicates that the LTE assumption is also justified for the M2-states for the parameters considered in this paper. Therefore all PPP-calculations have been carried out invoking LTE-populations although the code in principle can treat also non-LTE level populations. 


\section{RESULTS AND DISCUSSION}

\section{A. Calculations: $K^{2} L^{7} M^{1}$-configuration}

The first set of Stark broadening calculations involves transitions of type $K^{2} L^{7} M^{1} \rightarrow K^{2} L^{8}+h \nu$ and $K^{2} 2 s^{1} 2 p^{6} M^{1} \rightarrow K^{2} 2 s^{2} 2 p^{5} M^{1}+h \nu$. The first type of these transitions has a very low photon relaxation time (order of $0.1-1 \mathrm{~ns}$ ) and is therefore also sensitive to the long lasting recombination regime. Time integrated spectra might therefore show a much lower average temperature than the M2-states [9]. We therefore performed simulations also for temperature down to $8 \mathrm{eV}$ for the M1-states. The simulations involved all 36 states associated with the $K^{2} L^{7} M^{1}$-configuration that produced about 200 electric dipole transitions between 7.75 and $32.6 \mathrm{~nm}$. The ionic Stark effect has been taken into account for all the cases. When necessary 50 fields have been chosen to describe the ionic electric field distribution function obtained by the APEX method. This configuration allowed us to work with the whole atomic system when interference terms are taken into account. Typically, the run time on a single core of an Intel@ Xeon (R) CPU E5630 at $2.53 \mathrm{GHz}$ ranges between $30 \mathrm{sec}$ to $5 \mathrm{~min}$, depending on electron temperature and density. Fig. 3 shows the variation of the spectral distribution for fixed electron temperatures $\mathrm{T}_{e}=8,30 \mathrm{eV}$ but different electron densities $\mathrm{n}_{e}=10^{21}, 3 \times 10^{21}, 5 \times 10^{21} \mathrm{~cm}^{-3}$. Fig. 4 shows the same calculations but for fixed density and different temperatures. Solid lines include interference effects whereas dashed lines do not take into account interference effects.

Figs. 3 and 4, show that intra-shell L-L transition (around $26 \mathrm{~nm}$ ) are negligible compared to the Al IV lines (at 13.1 and $16 \mathrm{~nm}, \mathrm{M}-\mathrm{L}$ transitions) at about 8 $\mathrm{eV}$ electron temperature. At about $30 \mathrm{eV}$, the emission from $2 \mathrm{p} \rightarrow 2 \mathrm{~s}$ states starts to be important and is even more intense than M-L transitions. This is mainly due to the exponential factor in the Boltzmann populations because the ionization potential of the $2 \mathrm{~s}$ electrons are much larger than those of the $2 \mathrm{p}$ electrons. As Figs. 3 and 4 show, intra-shell transitions are negligible at $8 \mathrm{eV}$.

\section{B. Calculations: $K^{2} L^{7} M^{2}$-configuration}

The second set of calculations involves transitions of the type $\mathrm{K}^{2} \mathrm{~L}^{7} \mathrm{M}^{2} \rightarrow \mathrm{K}^{2} \mathrm{~L}^{8} \mathrm{M}^{1}+h \nu$ and $K^{2} 2 s^{1} 2 p^{6} M^{2} \rightarrow$ $K^{2} 2 s^{2} 2 p^{5} M^{2}+h \nu$. These transitions play an important role in the analysis as their time scale $\tau$ is determined by the large autoionizing rate $\Gamma$ (order of $10^{13}-10^{15} \mathrm{~s}^{-1}$ ) rather than the slow radiative decay rates $A$ (order of $10^{9}-10^{10} \mathrm{~s}^{-1}$ ) [23]:

$$
\tau=\frac{1}{\sum A+\sum \Gamma}=1-100 \mathrm{fs}
$$

Due to this fast time scale, the observed emission is related to the high density plasma just after the laser pulse: $\mathrm{T}_{e} \approx 25 \mathrm{eV}$ and $\mathrm{n}_{e} \approx 10^{22} \mathrm{~cm}^{-3}$ respectively [9].

The calculations of the $K^{2} L^{7} M^{2}$-configuration are rather complex: about 250 different energy levels have been considered, providing more than 5500 electric dipole transitions in the range between 7.75 and $32.6 \mathrm{~nm}$. The large number of transitions has a critical impact on the run time: we optimized the number of field and transition groups to give a solution in a reasonable amount of computational time. First, Stark ionic effect has been taken into account by using a unique ionic electric field set to the mean value of the field distribution, as shown in Fig. 5. It has been checked that due to the huge number of Stark transitions involved in the profiles of interest, the main overall feature of the ionic Stark effect is well reproduced by using only one field. Second, 50 transitions have been grouped together to consider the overwhelming part responsible for interference effects while keeping a reasonable computer time. The run time was thus between 6-8 days per pair of temperature and densities on the same computer than used in section III A.

Fig. 6 shows the variation of the spectral distribution for fixed electron temperatures $\mathrm{T}_{e}=10,25,100 \mathrm{eV}$ but different electron densities $\mathrm{n}_{e}=5 \times 10^{21}, 10^{22}, 2 \times 10^{22}$ $\mathrm{cm}^{-3}$. Fig. 7 shows the same calculations but for fixed density and different temperatures. As before, solid lines include interference effects whereas dashed lines do not take into account interference effects.

We would like to emphasize that these calculations, for either $\mathrm{K}^{2} \mathrm{~L}^{7} \mathrm{M}^{1}$ and $\mathrm{K}^{2} \mathrm{~L}^{7} \mathrm{M}^{2}$ configurations, have been performed assuming the electrons to be a non-degenerate gaz. To account for more extreme state of matter, where the temperature is lower than those used in our calculations, for example, the populations need to be described by a Fermi-Dirac distribution, which significantly increases the complexity of the atomic model.

\section{Discussion on interference effects}

Calculations taking into account interference terms for $\mathrm{K}^{2} \mathrm{~L}^{7} \mathrm{M}^{1}$-configuration (solid lines in Figs. 3 and 4 ) show an important behaviour: interference terms strongly affect the intra-shell L-L transitions while leaving almost unaffected the M-L transitions. As can be seen from the figures, the interference effects lead to a considerable narrowing of the L-L emission group near $26 \mathrm{~nm}$. For configuration of the type $\mathrm{K}^{2} \mathrm{~L}^{7} \mathrm{M}^{2}$ similar effects are observed. Fig. 6 and 7 show a strong line narrowing for L-L transitions while M-L transitions remain almost unaffected by interference effects.

The difference in contributions of the interference effects between the resonance M-L and intra-shell L-L transitions is based on their respective electronic configuration. The line broadening increases as $n^{4}$ where $n$ is the principal quantum number of the excited electron responsible for the emitted line. In the particular case where 


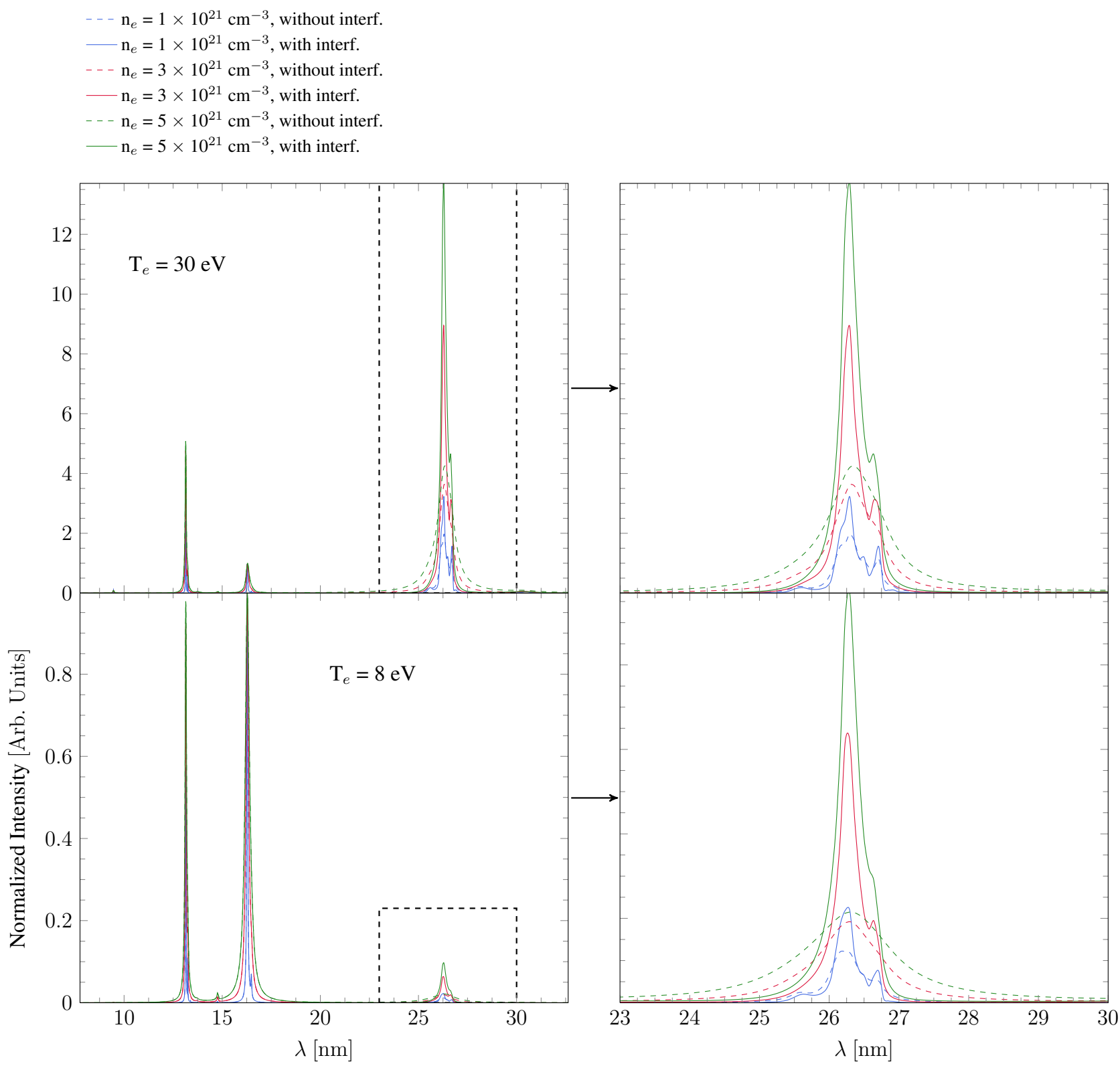

FIG. 3. Stark broadening of transitions originating from $\mathrm{Al} \mathrm{K} \mathrm{K}^{2} \mathrm{M}^{1}$ configuration for electron temperatures of $\mathrm{T}_{e}=8 \mathrm{eV}$ (bottom), $\mathrm{T}_{e}=30 \mathrm{eV}$ (top) and densities of $\mathrm{n}_{e}=10^{21} \mathrm{~cm}^{-3}$ (blue), $\mathrm{n}_{e}=3 \times 10^{21} \mathrm{~cm}^{-3}$ (red), $\mathrm{n}_{e}=5 \times 10^{21} \mathrm{~cm}^{-3}(\mathrm{green})$. Curves are plain for calculations with interference terms and dashed when not taken into account. Each curve is normalized to the peak intensity of the transitions around $16 \mathrm{~nm}$ for comparison purpose. A zoom of regions delimited by black dash rectangles is shown on the right side. 
there are several excited electrons, each excited electron (optical or spectator electron) contributes to the relaxation process and its contribution is more or less important depending on $n$. Moreover, the interactions between the optical and spectator electrons result into a splitting of the no-interaction line into a set of overlapping transitions which are dynamically coupled together by the embedding plasma. Ignoring this coupling (or interference terms) to calculate the line broadening will result into a sum of overlapping Lorentzians whose width will depend on $n$ and the electronic density $n_{e}$. Accounting for the dynamic coupling leads to a mixing and a coalescence of the overlapping transitions. This effect results from a compromise between the splitting width and the degree of overlapping of the lines involved in the splitting pattern.

In the present study, considering the intra-shell transitions, the contribution of the spectator electron for which $n=3$ is much more important than that of the active electron for which $n=2$. The dynamical interferences lead to a reduction of the spectator electron effects. In the high density limit the split transitions coalesce to the no-interaction line, and the broadening tends to the $n=2$ limit instead of $n=3$. According to this remark, one can infer that the resonant transitions $1 s^{2} 2 s^{2} 2 p^{5} 3 l \rightarrow 1 s^{2} 2 s^{2} 2 p^{6}$ for which there is no spectator electron beside the $n=3$ active electron, will show no interference effects. Concerning the satellite lines $1 s^{2} 2 s^{2} 2 p^{5} 3 l 3 l^{\prime} \rightarrow 1 s^{2} 2 s^{2} 2 p^{6} 3 l^{\prime}$, a $n=3$ spectator electron is present beside the $n=3$ active electron but the interference effects are minimized as the two excited electrons have a similar contribution to broadening. The effects of the interference terms on the intra-shell transitions are very strong and they increase with the density. They are stronger for the $\mathrm{K}^{2} \mathrm{~L}^{7} \mathrm{M}^{1}$ configuration than for the $\mathrm{K}^{2} \mathrm{~L}^{7} \mathrm{M}^{2}$ configuration as for the latter the splitting pattern is larger due to the presence of two spectator electrons. Thus, for the same density (for the same magnitude of the coupling terms) the line mixing effects are smaller. According to this remark and comparing the importance of the line narrowing in both cases for the same density, make us confident in the approximation made to calculate the $\mathrm{K}^{2} \mathrm{~L}^{7} \mathrm{M}^{2}$ configuration.

This observation allows direct studying of interference effects under real experimental dense plasma conditions: as L-L and M-L transitions in aluminum can be simultaneously observed with a single grating spectrometer, simulations with/without interference effects can directly be compared with the data. It is important to point out that the M-L transitions serve as calibration lines (that are almost of similar shape with/without interference effects). If only L-L transitions can be observed, an independent density diagnostic is requested which is extremely difficult to realize under real experimental conditions at FELs. Fig. 8 shows the general trend of the ratio between the intensity maximum of intra-shell L-L lines around $26 \mathrm{~nm}$ that include interference terms over the intensity of the same transitions without the interference terms. In the graph on the left, that shows the ratio with respect to the electron density for 3 different temperatures, we observe almost straight lines. The graph on right of Fig 8 shows the same ratio with respect to temperature. The large ratios clearly show the importance of interference effects: it does not lead to some corrections to the overall line shape but changes entirely the spectral distribution and even relative line ratios up to factors of about 4 .

\section{Quasi-continuous emission}

As can be seen from Figs. 4 and 7 the line broadening related to the configuration $\mathrm{K}^{2} \mathrm{~L}^{7} \mathrm{M}^{2}$ is much more pronounced compared to the $\mathrm{K}^{2} \mathrm{~L}^{7} \mathrm{M}^{1}$ configuration. The additional screening electron increases the number of atomic levels resulting in a higher sensitivity to plasma electric fields. Fig. 7 shows, that at densities larger than about $10^{22} \mathrm{~cm}^{-3}$ a quasi continuum forms. Depending on the electron temperature the continuum slope might be considered being positive or negative. These observations will have important impact when extracting the electron temperature from the continuum radiation (Bremsstrahlung or Planck radiation).

\section{CONCLUSION}

Complex line broadening simulations have discovered important line narrowing effects for intra-shell transitions due to interference effects. The simultaneous consideration of M-L and L-L transitions shows that M-L transitions are almost unaffected. This suggests that both the simultaneous observation of the resonant and intrashell transitions can be used to study the interference effects in FELs/matter interaction. The study of $\mathrm{K}^{2} \mathrm{~L}^{7} \mathrm{M}^{1}$ and $\mathrm{K}^{2} \mathrm{~L}^{7} \mathrm{M}^{2}$ configurations revealed that line broadening and interference effects show up at much lower densities for M2 compared to M1. A further advantageous property to employ M2-configuration for the analysis of data is that high auto-ionizing rates of the multiple excited M2-levels reduce the characteristic photon emission time scale down to fs scale.

\section{ACKNOWLEDGMENTS}

Supports from the projects "Émergence-2010: Métaux transparents créés sous irradiations intenses émises par un laser XUV/X à électrons libre" of the Pierre et Marie Curie University and the "Extreme Matter Institut EMM" are greatly appreciated. 


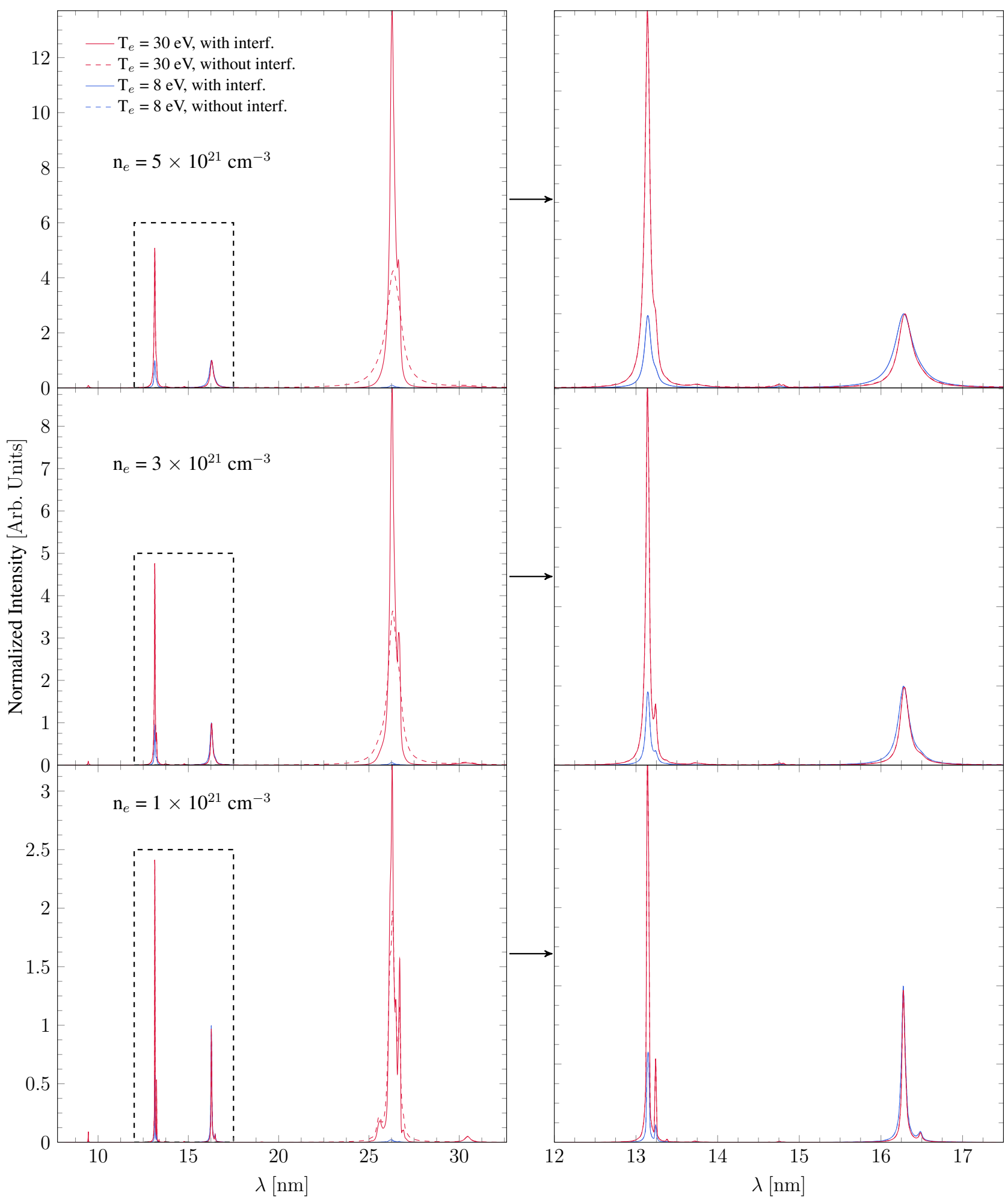

FIG. 4. Stark broadening of transitions originating from $\mathrm{Al} \mathrm{K}^{2} \mathrm{~L}^{7} \mathrm{M}^{1}$ configuration for electron densities of $\mathrm{n}_{e}=10^{21} \mathrm{~cm}^{-3}$ (bottom), $\mathrm{n}_{e}=3 \times 10^{21} \mathrm{~cm}^{-3}$ (middle), $\mathrm{n}_{e}=10^{22} \mathrm{~cm}^{-3}$ (top) and temperatures of $\mathrm{T}_{e}=8 \mathrm{eV}$ (blue), $\mathrm{T}_{e}=30 \mathrm{eV}$ (red). Curves are plain for calculations with interference terms and dashed when not taken into account. Each curve is normalized to the peak intensity of the transitions around $16 \mathrm{~nm}$ for comparison purpose. A zoom of regions delimited by black dash rectangles is shown on the right side. 


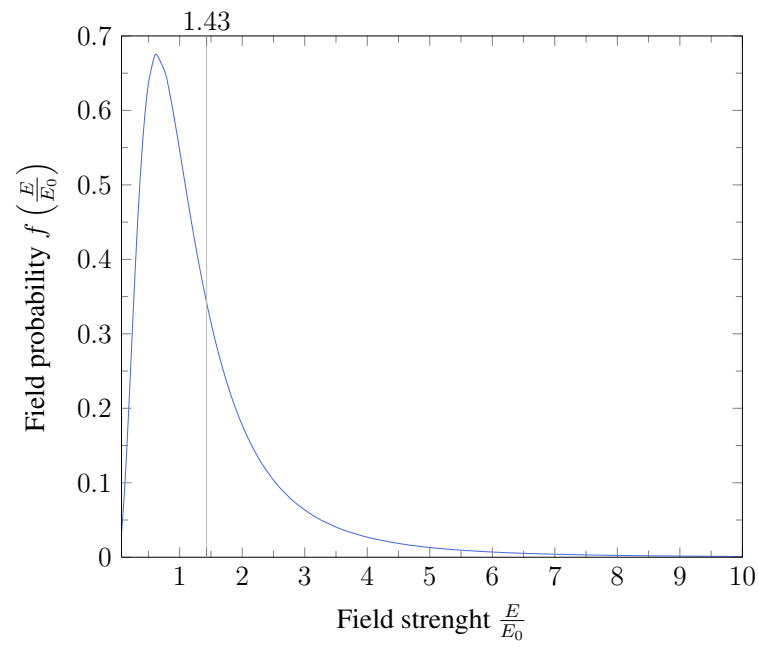

FIG. 5. Field distribution calculated using the APEX method $[16,17]$ for $\mathrm{K}^{2} \mathrm{~L}^{7} \mathrm{M}^{2}$-configuration at the electronic temperature and density of $\mathrm{T}_{e}=25 \mathrm{eV}$ and $\mathrm{n}_{e}=10^{22} \mathrm{~cm}^{-3}$ respectively. Values are normalized to the mean electronic field $E_{0}$. In this example, the mean value of 1.43 is used and describes the single field that runs the calculation with interference terms instead of the full distribution (blue curve).

[1] W. B. Hubbard, "Interiors of the giant planets," Science 214, 145-149 (1981).

[2] H. M. Van Horn, "Dense astrophysical plasmas," Science 252, 384-389 (1991)

[3] John Lindl, "Development of the indirect-drive approach to inertial confinement fusion and the target physics basis for ignition and gain," Phys. Plasmas 2, 3933-4024 (1995).

[4] Richard W. Lee, Stephen J. Moon, Hyun-Kyung Chung, Wojciech Rozmus, Hector A. Baldis, Gianluca Gregori, Robert C. Clauble, Otto L. Landen, Justin S. Wark, Andrew Ng, Steven J. Rose, Ciaran L. Lewis, Dave Riley, Jean-Claude Gauthier, and Patrick Audebert, "Finite temperature dense matter studies on next-generation light sources," J. Opt. Soc. Am. B 20, 770 (2003).

[5] F.B. Rosmej and R.W. Lee, "Hollow ion emission driven by pulsed x-ray radiation fields," Europhysics Letters 77, 24001 (2007).

[6] F.B. Rosmej, Exotic states of high density matter driven by intense XUV/X-ray Free Electron Lasers, edited by S. Varró (InTech, 2012) pp. 187-212.

[7] B. Nagler, U. Zastrau, R.R. Fäustlin, S.M. Vinko, T. Whitcher, A. J. Nelson, R. Sobierajski, J. Krzywinski, J. Chalupsky, E. Abreu, S. Bajt, T. Bornath, T. Burian, H. Chapman, J. Cihelka, T. Döppner, S. Düsterer, T. Dzelzainis, M. Fajardo, E. Förster, C. Fortmann, E. Galtier, S. H. Glenzer, S. Göde, G. Gregori, V. Hajkova, P. Heimann, L. Juha, M. Jurek, F. Y. Khattak, A. R. Khorsand, D. Klinger, M. Kozlova, T. Laarmann, H. J. Lee, R. W. Lee, R. H. Meiwes-Broer, P. Mercere, W. J. Murphy, A. Przystawik, R. Redmer, H. Reinholz, D. Riley, G. Röpke, F. B. Rosmej, K. Saksl
R. Schott, R. Thiele, J. Tiggesbäumker, S. Toleikis, T. Tschentscher, I. Uschmann, H. J. Vollmer, and J. S. Wark, "Turning solid aluminium transparent by intense soft x-ray photoionization," Nat. Phys. 5, 693-696 (2009).

8] L. Young, E. P. Kanter, B. Krässig, Y. Li, A. M. March, S. T. Pratt, R. Santra, S. H. Southworth, N. Rohringer, L. F. DiMauro, G. Doumy, C. A. Roedig, N. Berrah, L. Fang, M. Hoener, P. H. Bucksbaum, J. P. Cryan, S. Ghimire, J. M. Glownia, D. A. Reis, J. D. Bozek, C. Bostedt, and M. Messerschmidt, "Femtosecond electronic response of atoms to ultra-intense x-rays," Nature 466, 56-61 (2010).

[9] E. Galtier, F. B. Rosmej, D. Riley, T. Dzelzainis, F. Y. Khattak, P. Heimann, R. W. Lee, S. M. Vinko, T. Whitcher, B. Nagler, A. J. Nelson, J. S. Wark, T. Tschentscher, S. Toleikis, R. Fäustlin, R. Sobierajski, M. Jurek, L. Juha, J. Chalupsky, V. Hajkova, M. Kozlova, and J. Krzywinski, "Decay of crystalline order and equilibration during solid-to-plasma transition induced by 20 -fs microfocused 92 ev free electron laser pulses," Phys. Rev. Lett. 106, 164801 (2011).

[10] A. Calisti, F. Khelfaoui, R. Stamm, B. Talin, and R. W. Lee, "Model for the line shapes of complex ions in hot and dense plasmas," Phys. Rev. A 42, 5433-5440 (1990).

[11] A. Calisti, C. Mossé, S. Ferri, B. Talin, F. Rosmej, L. A. Bureyeva, and V. S. Lisitsa, "Dynamic stark broadening as the dicke narrowing effect," Phys. Rev. E 81, 016406 (2010).

[12] I.I. Sobelman, L.A. Vainshtein, and E.A. Yukov, Excitation of Atoms and Broadening of Spectral Lines, edited by New York Berlin, Heidelberg, Vol. 7 (Springer, 1981). 

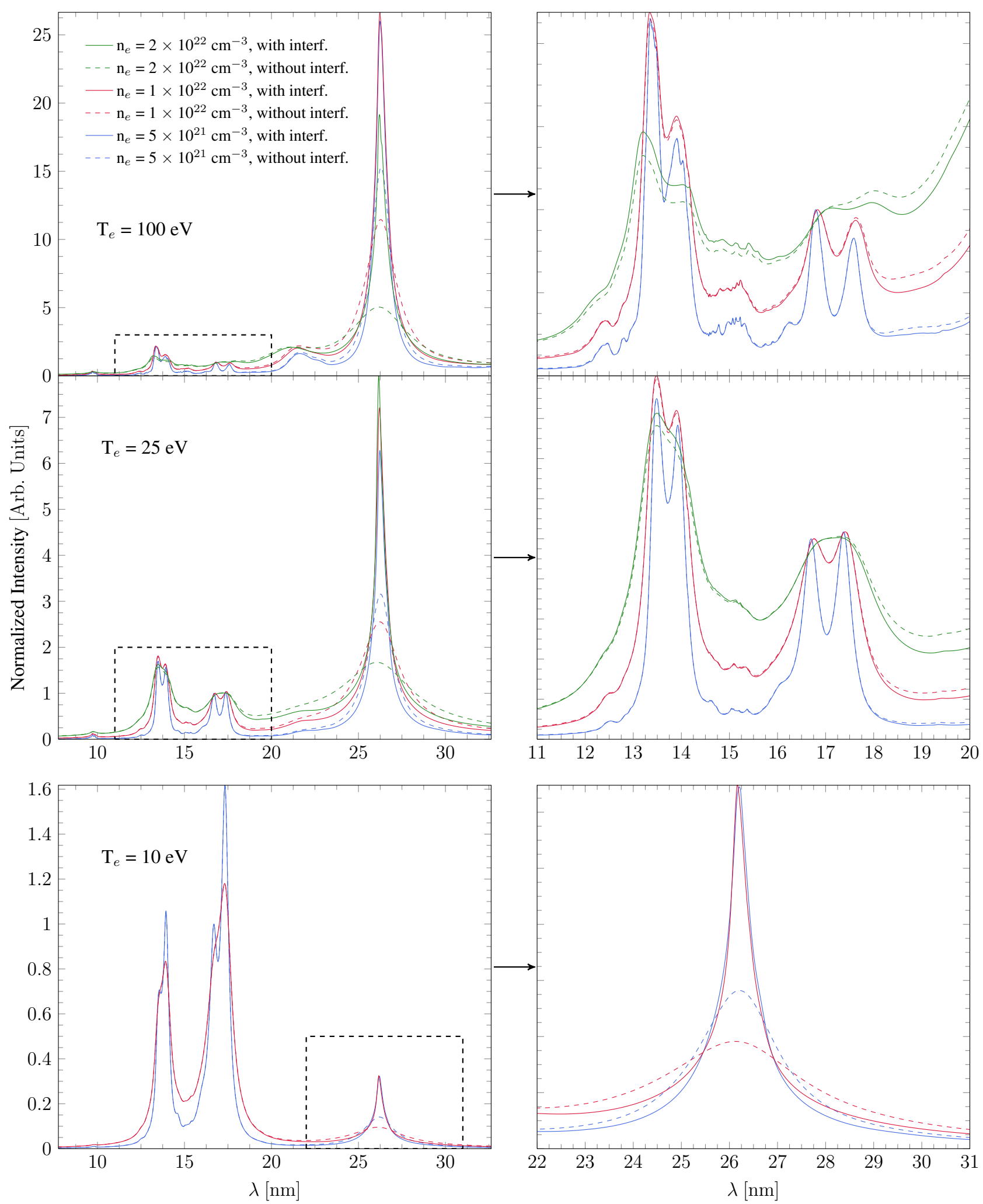

FIG. 6. Stark broadening of transitions originating from $\mathrm{Al} \mathrm{K}^{2} \mathrm{~L}^{7} \mathrm{M}^{2}$ configuration for electron temperatures of $\mathrm{T}_{e}=10 \mathrm{eV}$ (bottom), $\mathrm{T}_{e}=25 \mathrm{eV}$ (middle), $\mathrm{T}_{e}=100 \mathrm{eV}$ (top) and densities of $\mathrm{n}_{e}=5 \times 10^{21} \mathrm{~cm}^{-3}$ (blue), $\mathrm{n}_{e}=10^{22} \mathrm{~cm}^{-3}$ (red), $\mathrm{n}_{e}=2$ $\times 10^{22} \mathrm{~cm}^{-3}$ (green). Curves are plain for calculations with interference terms and dashed when not taken into account. Each curve is normalized to the peak intensity of the transitions around $16 \mathrm{~nm}$ for comparison purpose. A zoom of regions delimited by black dash rectangles is shown on the right side. 
[13] L. Godbert-Mouret, T. Meftah, A. Calisti, R. Stamm, B. Talin, M. Gigosos, V. Cardenoso, S. Alexiou, R. W. Lee, and L. Klein, "Accuracy of stark broadening calculations for ionic emitters," Phys. Rev. Lett. 81, 55685571 (1998)

[14] Carlos A. Iglesias, "Excited spectator electron effects on spectral line shapes," High Energy Density Physics 6, 318-331 (2010).

[15] Hans R. Griem, Principles of Plasma spectroscopy, edited by M. G. Haines, Cambridge Monographs on Plasma Physics (Cambridge University Press, 2005).

[16] Carlos A. Iglesias, Hugh E. DeWitt, Joel L. Lebowitz, David MacGowan, and William B. Hubbard, "Lowfrequency electric microfield distributions in plasmas," Phys. Rev. A 31, 1698-1702 (1985).

[17] C.A. Iglesias, F.J. Rogers, R. Shepherd, A. Bar-Shalom, M.S. Murillo, D.P. Kilcrease, A. Calisti, and R.W. Lee, "Fast electric microfield distribution calculations in extreme matter conditions," J. Quant. Spectrosc. Radiat. Transfer 65, 303-315 (2000).

[18] Earl W. Smith and C. F. Hooper, "Relaxation theory of spectral line broadening in plasmas," Phys. Rev. 157, 126-137 (1967).

[19] Hans R. Griem, Milan Blaha, and Paul C. Kepple, "Stark-profile calculations for lyman-series lines of oneelectron ions in dense plasmas," Phys. Rev. A 19, 24212432 (1979)

[20] Ming Feng Gu, "The flexible atomic code," Canadian Journal of Physics 86, 675-689 (2008).

[21] F.B. Rosmej, F. Petitdemange, and E. Galtier, "Identification of Auger electron heating and inverse Auger effect in experiments irradiating solids with XUV free electron laser radiation at intensities larger than $10^{16} \mathrm{~W} / \mathrm{cm}^{2}$," in Proceedings of the SPIE International Symposium on Optical Engineering and Applications " $X$-Ray Lasers and Coherent X-Ray Sources: Development and Applications (OP321) " (2011) paper 8240-2.

[22] F. Petitdemande and F. B. Rosmej, "Dielectronic satellites and auger electron heating : irradiation of solids by intense xuv-free electron laser radiation," in New Trends in Atomic 83 Molecular Physics - Advanced Technological Applications, edited by M. Mohan (Springer, 2013) pp. $1-28$, in print.

[23] F.B. Rosmej, X-ray emission spectroscopy and diagnostics of non-equilibrium fusion and laser produced plasmas, edited by Y. Zou and R. Hutton (Taylor and Francis, 2012) pp. 267-341.

[24] U. Zastrau, C. Fortmann, R. R. Fäustlin, L. F. Cao, T. Döppner, S. Düsterer, S. H. Glenzer, G. Gregori, T. Laarmann, H. J. Lee, A. Przystawik, P. Radcliffe, H. Reinholz, G. Röpke, R. Thiele, J. Tiggesbäumker, N. X. Truong, S. Toleikis, I. Uschmann, A. Wierling, T. Tschentscher, E. Förster, and R. Redmer, "Bremsstrahlung and line spectroscopy of warm dense aluminum plasma heated by xuv free-electron-laser radiation," Phys. Rev. E 78, 066406 (2008).

[25] Jochen R. Schneider, "FLASH-from accelerator test facility to the first single-pass soft x-ray free-electron laser," J. Phys. B: Atom. Mol. Opt. Phys. 43, 194001 (2010).

[26] J. Feldhaus, "FLASH-the first soft x-ray free electron laser (FEL) user facility," J. Phys. B: Atom. Mol. Opt. Phys. 43, 194002 (2010). 


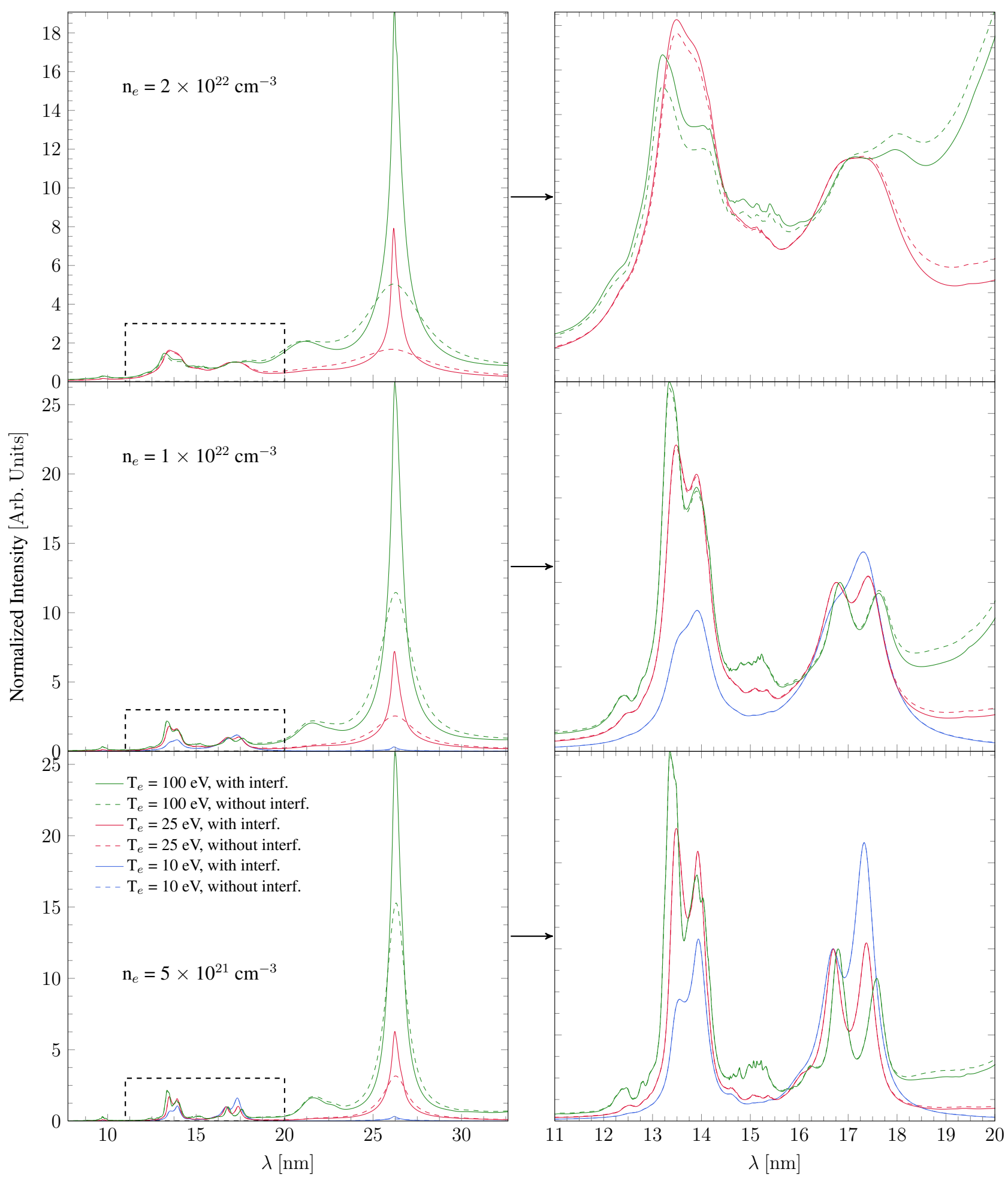

FIG. 7. Stark broadening of transitions originating from $\mathrm{Al} \mathrm{K}^{2} \mathrm{~L}^{7} \mathrm{M}^{2}$ configuration for electron densities of $\mathrm{n}_{e}=5 \times 10^{21}$ $\mathrm{cm}^{-3}$ (bottom), $\mathrm{n}_{e}=10^{22} \mathrm{~cm}^{-3}$ (middle), $\mathrm{n}_{e}=2 \times 10^{22} \mathrm{~cm}^{-3}$ (top) and temperatures of $\mathrm{T}_{e}=10 \mathrm{eV}$ (blue), $\mathrm{T}_{e}=25 \mathrm{eV}$ (red), $\mathrm{T}_{e}=100 \mathrm{eV}$ (green). Curves are plain for calculations with interference terms and dashed when not taken into account. Each curve is normalized to the peak intensity of the transitions around $16 \mathrm{~nm}$ for comparison purpose. A zoom of regions delimited by black dash rectangles is shown on the right side. At near solid density, quasi-continuum signal is observed for transitions originating from the multiple excited states $\mathrm{K}^{2} \mathrm{~L}^{7} \mathrm{M}^{2}$. 


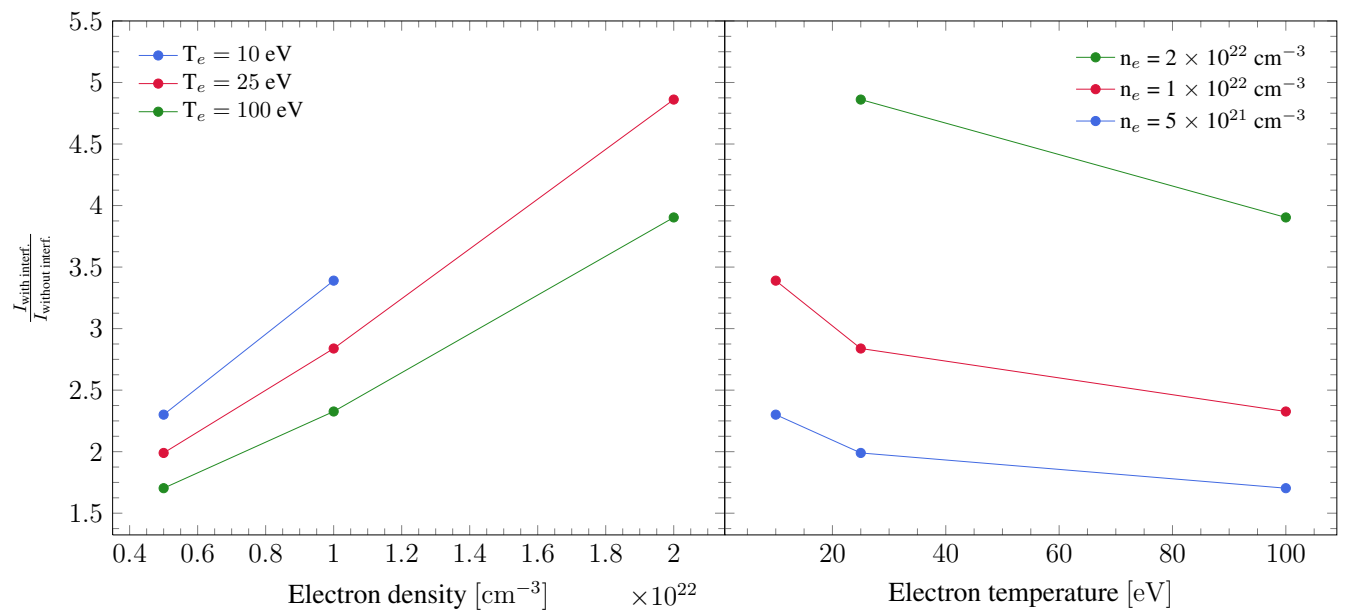

FIG. 8. Ratio of the group maximum intensity of $M^{2}$ intra-shell transitions calculated without and with interference effects taking into account the dependence in density (left) and temperature (right). 